\title{
Las cartas de servicios y los premios a la calidad en la administración general del Estado español ${ }^{1}$
}

\author{
Amador Elena Córdoba
}

\section{Antecedentes}

La evolución de la sociedad y la transformación del contexto en que se desenvuelven las organizaciones que prestan servicios exigen una renovación de la Administración. Precisamente, la prestación de servicios constituye un elemento central de las reformas en la mayor parte de los países de nuestro entorno.

Se trata de poner la "Administración al servicio del público" como ya señalaba la OCDE en 1987, puesto que el ciudadano espera de la Administración Pública:

- que el sistema administrativo sea comprensible;

- que responda a sus necesidades;

- que su relación con la Administración sea en condiciones accesibles;

- que pueda participar más activamente en la gestión administrativa.

Por ello, en el ámbito de la Unión Europea y de la OCDE, las Administraciones nacionales vienen incluyendo en sus procesos de reforma la "gestión de calidad" como una opción para avanzar en la mejora de la gestión pública y satisfacer las demandas ciudadanas.

La "gestión de calidad"está orientada a los usuarios, a la obtención de resultados acordes con lo que ellos esperan, y se basa en la mejora continua de la prestación del servicio, a partir del análisis del cumplimiento de objetivos y la evaluación de los resultados obtenidos y de la satisfacción de los propios usuarios.

En la Ley 6/1997, de 14 de abril, de Organización y Funcionamiento de la Administración General del Estado (LOFAGE), ya se afirma que:

Amador Elena

Córdoba es

Director

General de Inspección,

Simplificación

y Calidad de los

Servicios del

Ministerio de

Administraciones

Públicas (MAP),

España.

Contato:

publicaciones @ inap.map.es 
- "el servicio a los ciudadanos es el principio básico que debe presidir la actividad administrativa";

y también que

- "los ciudadanos tienen el legítimo derecho a saber cuáles son las competencias de cada Administración y a recibir servicios públicos de calidad".

La LOFAGE orienta la gestión de los servicios al ciudadano, a través de los principios de organización y funcionamiento de:

- "simplicidad, claridad y proximidad a los ciudadanos", y

- "el servicio efectivo a los ciudadanos".

La LOFAGE dedica un apartado especial al "principio de servicio a los ciudadanos", estableciéndose que la actuación de la Administración General del Estado (AGE) debe asegurar a los ciudadanos:

- una continua mejora de los procedimientos, servicios y prestaciones públicos de acuerdo con las políticas fijadas por el Gobierno y teniendo en cuenta los recursos disponibles;

- determinando al respecto las prestaciones que proporcionan los servicios estatales, sus contenidos y los correspondientes estándares de calidad.

Por todo ello, el MAP, dentro de sus actuaciones encaminadas a promover la mejora continua de los servicios públicos, y con el fin de desarrollar los contenidos de la LOFAGE, ha impulsado la adopción por parte del Consejo de Ministros del Real Decreto 1259/1999 (Boletín Oficial del Estado del 10 de agosto de 1999), para regular las Cartas de Servicios y establecer los Premios a la Calidad y a las Mejores Prácticas.

Por otra parte, el Real Decreto establece un marco general para la evaluación y mejora de los servicios prestados por la Administración General del Estado fundamentado en el internacionalmente reconocido Modelo Europeo de Autoevaluación de la Calidad, de la Fundación Europea para la Gestión de Calidad (EFQM). Este modelo de referencia permite realizar una evaluación consistente de las organizaciones y su comparación en términos homogéneos.

\section{Contenido}

\section{- Objeto y ámbito de aplicación}

El Real Decreto define su finalidad de regular las Cartas de Servicios, el marco para la evaluación y los Premios a la Calidad como instrumentos de desarrollo del principio de servicio a los ciudadanos establecido en la LOFAGE. Se delimita el ámbito de aplicación a la Administración General del Estado, lo que incluye a sus Órganos y a los 
Organismos Autónomos vinculados o dependientes de la misma. No obstante, la Disposición adicional única del Real Decreto prevé la aplicación voluntaria de sus normas por parte de otros Organismos públicos como las entidades públicas empresariales o la Agencia Estatal de Administración Tributaria.

\section{- Definición, contenido y estructura de las Cartas de Servicios}

El artículo 4.1 de la LOFAGE, que regula el "principio de servicio a los ciudadanos" dispone que para la mejora de los servicios que presta la Administración General del Estado se determinarán "las prestaciones que proporcionan los servicios estatales, sus contenidos y los correspondientes estándares de calidad".

Para esa determinación, se elabora un Real Decreto que se inspira en el principio de mejora continua de los servicios públicos en función de las demandas ciudadanas.

El Real Decreto regula las "Cartas de Servicios" y profundiza en:

- la transparencia;

- la información;

- la participación y consulta a los usuarios, y

- la responsabilización de los gestores públicos.

El objetivo de las "Cartas de Servicios" es traducir las expectativas ciudadanas en mejoras de los servicios de la AGE, a través:

- del suministro de información cabal sobre la naturaleza, contenido, características y formas de los servicios;

- sobre sus estándares o niveles de prestación, y

- sobre los sistemas de participación, seguimiento y actualización de los compromisos contraídos por la Administración.

Las "Cartas de Servicios" hacen posible que:

- la organización administrativa se adapte a las necesidades de los ciudadanos, y que

- los ciudadanos estén en condiciones de conocer por anticipado qué clase de servicio pueden esperar y demandar, y cuáles son los compromisos de calidad del servicio.

Partiendo de la premisa de que no se establece un modelo cerrado y único, las Cartas se caracterizan como documentos divulgativos destinados al público en general para informarle sobre los aspectos de interés tanto intrínsecos como formales y facilitadores de las prestaciones y servicios a que pueden tener derecho. Asimismo, se determinan las reglas básicas de ordenación, estilo y presentación de la informacióm para que, sin detrimento del margen de libertad adecuado a las características de cada Órgano u Organismo, se garantice la fácil y correcta comprensión 
de su contenido por cualquier usuario o ciudadano, que es el destinatario de estos documentos. En este sentido, se concretan los elementos mínimos que deben contener todas las Cartas, como son:

a) La identificación y la descripción sucinta de los fines o misión del Órgano u Organismo, para facilitar desde el principio al ciudadano la identificación entre sus necesidades y la instancia competente para atendérselas.

b) Una relación explicativa o catálogo de las diferentes prestaciones y servicios que se proveen, especificando, en su caso, cuantas modalidades puedan revestir. Se trata con ello de que el usuario conozca detalladamente la oferta de servicios, con sus características, condiciones, extensión etc.

c) La forma de plantear quejas, reclamaciones y sugerencias, explicitando la disponibilidad y acceso al Libro de Quejas y Sugerencias.

d) La mención expresa a las fórmulas o mecanismos de participación o colaboración de los usuarios más adecuados al caso (cuestionarios de opinión, comités consultivos u otros).

e) La relación claramente referenciada de la normativa reguladora de cada una de las prestaciones y servicios, que habrá de ser puesta al día periódicamente para facilitar el acceso a su regulación sustantiva y procedimental.

f) Una declaración de compromisos de calidad, determinando los estándares que expresen de forma cuantificada o susceptible de verificación la calidad de los servicios proporcionados. A estos efectos se plantean una serie de aspectos comunes como:

- los plazos de los procesos o tiempos de respuesta;

- los mecanismos de información y comunicación que permitan la relación fluida y personalizada del usuario con el prestador del servicio (existencia y localizacion de puestos de información y atención, sistemas de comunicación telefónicos o telemáticos para información y gestión de trámites etc.);

- la amplitud y flexibilidad de la oferta horaria de atención al público;

- aquellas otras facilidades específicas que cada Órgano/Organismo pueda brindar a sus usuarios para mejorar la calidad del servicio.

g) Los indicadores que se utilizarán para evaluar la calidad.

h) Otra información de caráter complementario, como la identificación y dirección de la Unidad responsable de la Carta, los datos divulgativos sobre los servicios gestionados que se consideren de interés para el público y el Directorio (postal, telefónico y telemático) de las oficinas o centros donde se presta cada uno de los servicios, con objeto de que sus potenciales usuarios puedan encaminarse al domicilio concreto donde tramitar o recibir cada prestación o servicio y evitar, así, visitas infructuosas. Cuando el Órgano/Organismo disponga, además de la sede central, de 
una red descentralizada de servicios (regionales, provinciales, locales), habrán de consignarse todas las direcciones.

\section{- Proceso de elaboración, aprobación y difusión. Responsabilidades}

$\mathrm{Al}$ tratarse de un programa de alcance global para el conjunto de la AGE, se combinan el impulso y coordinación de la Secretaría de Estado para la Administración Pública con la salvaguarda de la iniciativa y autonomía de los distintos Departamentos ministeriales. Así, se diseña una estructura funcional en la que el impulso, coordinación general y el informe preceptivo corresponden a la Secretaría de Estado para la Administración Pública, la coordinación departamental y la aprobación a los Subsecretarios, y la elaboración, difusión y gestión a los titulares de los órganos y organismos responsables de los respectivos servicios.

Es destacable que para subrayar el compromiso real de cada órgano u organismo se establece la obligación de publicar la resolución por la que se aprueba cada Carta en el Boletín Oficial del Estado. Esta publicidad oficial se hace sin perjuicio de que cada organización lleve a cabo las medidas divulgativas convenientes, tanto del texto completo de la Carta como a través de folletos, trípticos etc., siempre que se garantice su disponibilidad para cualquier usuario en todas las dependencias con atención al público.

\section{- Evaluación de la calidad}

El Real Decreto prevé, asimismo, el sistema-marco de evaluación de la calidad de los servicios públicos estatales de un modo sencillo y articulado en dos niveles: a) la evaluación externa, que proporcionará a los órganos rectores de la AGE la información integrada necesaria para la ordenación y la mejora de los servicios prestados a los ciudadanos y que suministrará a éstos información sobre el seguimiento de los niveles de calidad de los servicios, de modo que pueda ejercer mejor sus derechos y valorar con conocimiento de causa el funcionamiento real de los servicios públicos; b) la autoevaluación, que permitirá a los responsables de la gestión corregir deficiencias, implantar procesos de mejora y dar a conocer a los usuarios los resultados obtenidos con ello.

La evaluación se contempla, como se ha dicho, desde dos aspectos: por una parte, la autoevaluación que cada Órgano/Organismo debe realizar anualmente a través del análisis de sus procesos y sus resultados y, por otra, el seguimiento de su Carta de Servicios. La Secretaría de Estado para la Administración Pública, como órgano horizontal especializado, asegurará la homogeneidad de las autoevaluaciones para posibilitar la 
coherencia y comparabilidad de la información y, además, sobre la base de tal información, presentará anualmente un informe conteniendo la evaluación global de la calidad del conjunto de servicios de la AGE seleccionados. Esta evaluación global se complementará con medidas para conocer el estado de opinión de los ciudadanos sobre los servicios estatales (estudios de opinión nacionales etc.). Por su parte, los órganos correspondientes de cada Ministerio - normalmente las Subsecretarías - reciben la responsabilidad de efectuar la evaluación externa de los órganos/organismos de su ámbito y de enlace con la Secretaría de Estado para la Administración Pública.

\section{- Competencias de gestión}

La ejecución de las funciones de impulso, asesoramiento, informe, colaboración en la elaboración de las Cartas y confección de la evaluación global se asigna a la Dirección General de Inspeción, Simplificación y Calidad de los Servicios, Órgano directivo de la Secretaría de Estado para la Administración Pública, cuyas competencias y especialización lo hacen idóneo para ese papel.

\section{Los premios a las mejores prácticas y el premio a la calidad en la Administración General del Estado}

Finalmente, se crean y establecen los aspectos básicos de los Premios a las Mejores Prácticas y el Premio a la Calidad en la Administración General del Estado, como mecanismos formales para impulsar el reconocimiento de las mejores prácticas de gestión y el incremento de la calidad en línea con las numerosas iniciativas desarrolladas en distintos ámbitos: Premio Europeo a la Calidad en el Sector Público (EFQM), diversos Premios Nacionales (Reino Unido, Dinamarca, Portugal, Italia etc.) y, en España, el Premio a la Calidad en el Sector Público de la Comunidad de Madrid o el Premio a la Calidad en Educación del Ministerio de Educación y Cultura.

Las múltiples experiencias desarrolladas tanto en el ámbito internacional como en algumas Administraciones públicas españolas aconsejaban introducir una iniciativa que permitiera institucionalizar un instrumento general de reconocimiento a la mejora de la calidad y de comparación y aprendizaje mutuos, como es la convocatoria anual de unos Premios a las Mejores Prácticas y un Premio a la Calidad en la AGE. Por ello el Real Decreto instituye tales Premios, destinados los

primeros a galardonar iniciativas de mejora de la calidad, y el segundo a 
reconocer al órgano u organismo que se haya distinguido muy especialmente en el incremento de la calidad de sus servicios, mediante la mejora de los procesos y resultados de la organización enteramente considerada. Se determinan así las características, requisitos y efectos de los Premios, que habrán de ser desarrollados en las órdenes de convocatoria del Ministro de Administraciones Públicas (número de premios, propuestas, selección final, forma de evaluación, jurado, reconocimiento organizacional y personal etc.).

\section{- Implantación}

La implantación del proceso de elaboración de las Cartas de Servicios se ha previsto de manera progresiva en dos fases: una primera, de un mes, en la que el Subsecretario de cada Ministerio selecionará, por la relevancia pública de sus servicios, el/los Órgano/s u Organismo/s de su ámbito, cuyas Cartas de Servicios serán prioritarias. Una vez comunicado lo anterior a la Secretaría de Estado para la Administración Pública, en un plazo máximo de seis meses, los Órganos/Organismos así seleccionados elaborarán su Carta de Servicios, aprobándose y publicándose seguidamente por los Subsecretarios. La implantación generalizada se llevará a cabo en función de los resultados obtenidos en el período señalado.

\section{Apuntes}

Texto publicado originalmente en la Revista Iberoamericana de Administración Pública, 1999, n.3, jul/dic. INAP/MAP. Autorizado por el Instituto Nacional de Administración Pública, Madrid, España.
} 
O artigo apresenta a lei no 6/1997 de Organização e Funcionamento da Administração Geral do Estado (LOFAGE), na Espanha, que orienta a gestão dos serviços prestados aos cidadãos e estabelece a atuação da Administração Geral do Estado (AGE), assegurando a melhoria na prestação dos serviços ao cidadão, de acordo com as políticas fixadas e os recursos disponíveis, bem como o respeito aos padrões de qualidade dos serviços estatais.

\section{Las cartas de servicios y los premios a la calidad en la administración general del Estado español}

Amador Elena Córdoba

El artículo presenta la ley 6/1997, 14 de abril, de Organización y Funcionamiento de la Administración General del Estado (LOFAGE), en España, que orienta la gestión de los servicios prestados a los ciudadanos y establece la actuación de la Administración General del Estado (AGE), asegurándose la mejora en la prestación de los servicios al ciudadano, de acuerdo con las políticas fijadas y los recursos disponibles, bien como el respecto a los estándares de calidad de los servicios estatales.

\section{Service Charters and National General Administration quality awards in Spain \\ Amador Elena Córdoba}

The article presents the law 6/1997, for organization and functioning of the National General Administration (LOFAGE), in Spain, that provides guidance on how to manage citizen services and defines the line of action to be pursued by the National General Administration (AGE) to ensure improved citizen services, in conformity with policies in force and available resources, and the compliance to public service quality standards.

Ano 51

Número 4

Out-Dez 2000

Amador Elena
Córdoba es
Director
General de
Inspección,
Simplificación
y Calidad de los
Servicios del
Ministerio de
Administraciones
Públicas (MAP),
España.
Contato:
publicaciones@
inap.map.es


A Revista do Serviço Público é uma publicação da ENAP Escola Nacional de Administração Pública, voltada para a divulgação e debate de temas relacionados ao Estado, à Administração Pública e à gestão governamental. Procurando o aprimoramento permanente da revista, tanto no seu conteúdo quanto na apresentação gráfica, pedimos aos nossos colaboradores a observação das normas abaixo descritas.

\section{Normas para os colaboradores}

1. Os artigos, sempre inéditos no Brasil, devem conter em torno de 25 laudas de 20 linhas de 70 toques.

2. Os originais devem ser encaminhados ao editor, em disquete, em programa de uso universal, com uma cópia impressa. Usar apenas as formatações-padrão.

3. Cada artigo deve vir acompanhado de um resumo analítico em português, espanhol e inglês, de cerca de 150 palavras, que permita uma visão global e antecipada do assunto tratado.

4. Na primeira página do artigo, deve constar informação sobre formação e vinculação institucional do autor (em até duas linhas).

5. Notas, referências e bibliografia devem vir ao final do artigo, e não ao pé da página. Notas e referências, sendo o caso, devem vir devidamente numeradas.

6. Além de artigos, a revista receberá comunicações, notas informativas, notícias e relatórios conclusivos de pesquisas em desenvolvimento, com até 15 laudas. Resenhas de livros, em torno de 4 laudas, devem conter uma apresentação sucinta da obra e eventuais comentários que situem o leitor na discussão.

7. Os trabalhos que se adequarem à linha temática da revista serão apreciados pelo conselho editorial, que decidirá sobre a publicação com base em pareceres de consultores ad hoc.

8. Os originais enviados à Revista do Serviço Público não serão devolvidos. A revista compromete-se a informar os autores sobre a publicação ou não de seus trabalhos.

\section{Convite a resenhadores}

A Revista do Serviço Público convida todos os interessados em remeter resenhas de trabalhos publicados no Brasil e no exterior sobre Estado, Administração Pública e gestão governamental.

As resenhas devem ser originais e não exceder a cinco laudas datilografadas em espaço duplo com 20 linhas de 70 toques, e devem apresentar de modo sucinto a obra, com comentários que situem o leitor na discussão apresentada.

As resenhas devem ser enviadas em português, sem notas de rodapé, contendo o título completo e subtítulo do livro, nome completo do autor, local de publicação, editora e ano de publicação, bem como uma breve informação sobre a formação e vinculação institucional do resenhador (em até duas linhas), acompanhadas do respectivo disquete.

\section{Nota aos editores}

Pedimos encaminhar à Revista do Serviço Público exemplares de livros publicados, a fim de serem resenhados. Os resenhadores interessados receberão cópias dos livros enviados. 